\title{
Selective Assignment of Energy Transfer and Charge Separation Pathways in Reaction Centers by Pulse Polarized 2-D Photon Echo Spectroscopy
}

\author{
Benjamin P. Fingerhut ${ }^{1}$, Kochise Bennett ${ }^{1}$, Oleksiy Roslyak ${ }^{1}$, and Shaul Mukamel ${ }^{1}$ \\ ${ }^{1}$ Chemistry Department, University of California, Irvine, California 92697-2025, USA
}

\begin{abstract}
We present theoretical modelling of the nonlinear optical response of the bacterial reaction center incorporating electron and energy transfer on equal footing. Orthogonal polarized pulse sequences allow to dissect kinetic components in real space.
\end{abstract}

\section{Combined excitation energy transfer and charge separation in reaction centers}

The understanding of the ultrafast initial photosynthetic steps is crucial for the design of artificial photochemical devices. At the heart of photosynthesis are excitation energy transfer (EET) and charge separation (CS) within optimized reaction centers (RC) in bacteria and plants. The close coupling between both processed guarantees the near unity efficiency. Simulations on photosynthetic complexes commonly focus on EET based on the Frenkel exciton Hamiltonian [1]. Realistic models have to incorporate all aspects of CS and EET, like e.g. coulomb attraction of generated electronhole pairs.

We present simulations on a tight-binding model of the bacterial reaction center $B$. viridis, which incorporate polaron effects thus allowing to describe EET and CS. The combination of parallel and orthogonal polarized pulses sequences allows to selectively address the dipole moments in $\mathrm{RC}$. The signal contribution of parallel-aligned exciton transitions is suppressed, highlighting weaker signal features. This allows to dissect the initial 3ps charge separation component in bacterial RC from EET and the secondary sub-picosecond electron transfer.

\section{Tight-binding model of the bacterial reaction center $B$. viridis}

The developed model of combined EET and CS in RC relies on a tight-binding formulation of the electronic Hamiltonian in the monopole approximation. The creation of electron-hole pairs can occur located on two level molecules or between different sites (Fig. 1, left). The considered coulomb attraction between charge carriers guarantees the prober description of all considered states, i.e. the single- and bi-exciton manifold. The model reduces to the Frenkel exciton model if only on-site excitations are considered, we additionally consider the charge separated states of the strongly coupled special pair $\left(\mathrm{P}-\mathrm{BCl}_{\mathrm{M}}, \mathrm{P}-\mathrm{BCl}_{\mathrm{L}}\right)$ and within the active $\mathrm{L}$-branch $\left(\mathrm{BCl}_{\mathrm{L}}, \mathrm{BP}_{\mathrm{L}}\right)$ of the reaction center B. viridis (Fig.1, right). 


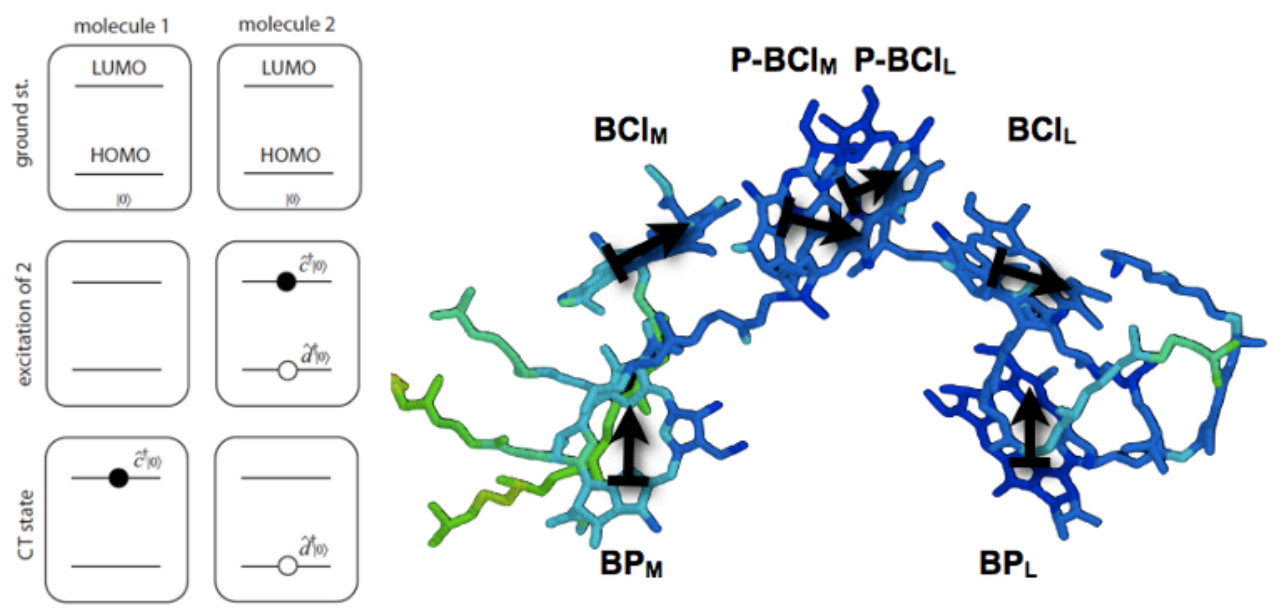

Fig. 1. On site tight-binding model (left) and considered chromophores in the reaction center of $B$. viridis. The orientation of site transition dipoles is indicated with black arrows.

Transport and relaxation between exciton states is induced bath fluctuations. We model the RC spectral density by coupling the electron and hole sites to a collection of harmonic bath modes, transport rates are calculated microscopically by modified Redfield theory [2]. The model reduces to the Marcus, Förster and Redfield limit and reproduces the temperature dependent absorption spectrum and charge transfer kinetics in the active L-branch.

\section{Linear combination of 2D photon echo signals}

We present the non-linear response of the RC model subject to broadband and phase stable pulses. Two different 2D photon-echo (2D-PE) signals are considered to complementarily discriminate between the kinetic components in the RC: first all pulses irradiate the sample in an all-parallel pulse configuration $(\mathrm{AP}=\mathrm{XXXX})$. The second cross-polarized signal (CP) consists of a linear combination of two of the three independent tensor components of the AP signal ( $\mathrm{CP}=\mathrm{XXYY}$ $\mathrm{XYXY}$ ), and is within reach of experiments [3]. The CP signal monitors the symmetry breaking between the stimulated emission and ground state bleach Feynman diagrams as the waiting time $t_{2}$ is increased, highlighting population transport between excitons.

\section{Charge separation dynamics monitored by pulse polarized 2D photon-echo spectroscopy}

In Figure 2 we present the simulated 2D-PE spectra of the $\mathrm{RC}$ model of $B$. viridis. The $\mathrm{CP}$ signal reveals additional features which are assigned to $\mathrm{P}^{-}-\mathrm{BP}_{\mathrm{L}}$ and $\mathrm{BCl}_{\mathrm{L}}-\mathrm{BP}_{\mathrm{L}}$ resonances. The analysis of the population dynamics (Fig. 2, right) shows that in the AP configuration sub-400 fs energy relaxation and subsequent 3 ps CS kinetics show up on the off-diagonal (crosspeaks $\mathrm{BCl}_{\mathrm{L}, \mathrm{ESA}}$ and $\mathrm{BCl}_{\mathrm{L}}{ }^{-}$). The population dynamics of the CS is evident in the bleach recovery of special pair $\mathrm{P}-$ of the 2D-PE for $t_{2}>1 \mathrm{ps}$. 
(a)
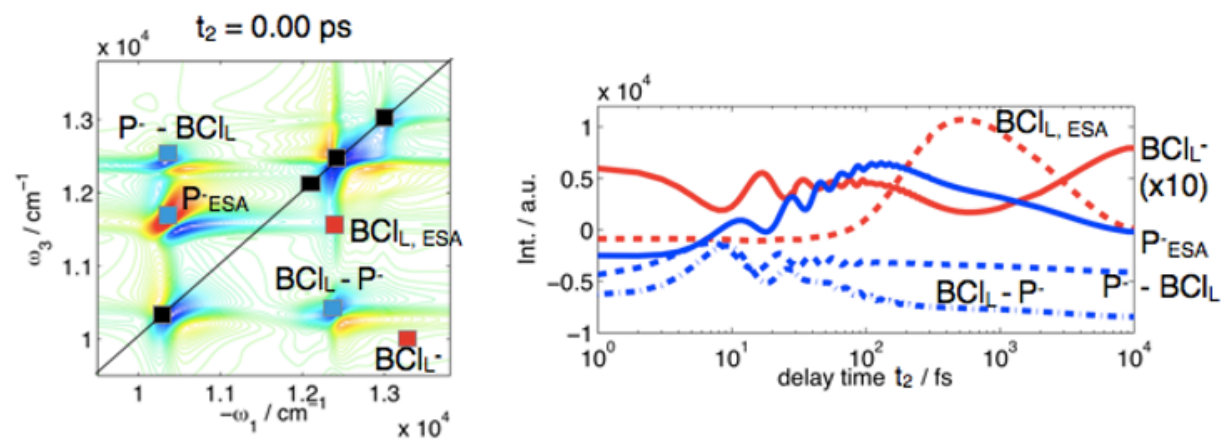

(b)
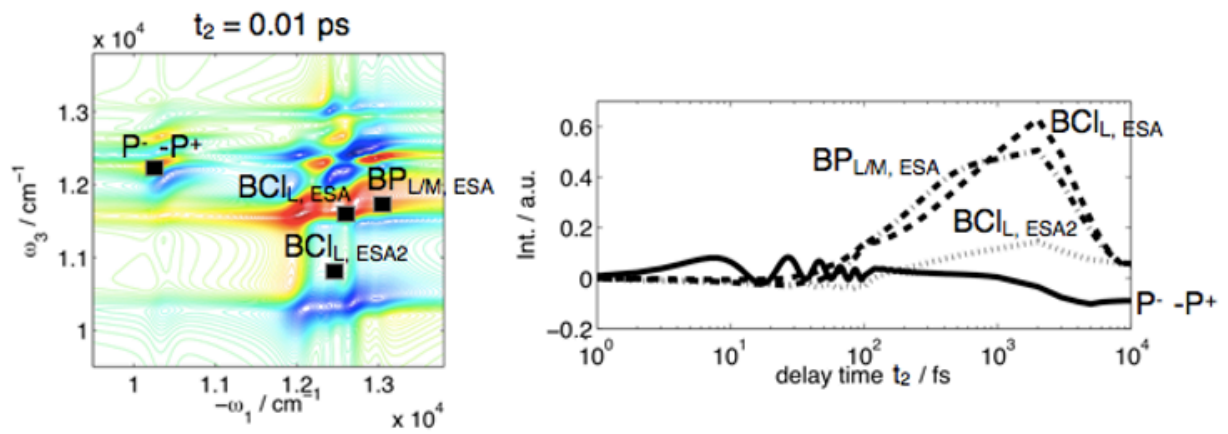

Fig. 2. Simulated 2D photon-echo spectra of the RC B. viridis in the AP pulse configuration (a) and the cross polarized signal $\mathrm{CP}=\mathrm{XXYY}-\mathrm{XYXY}(\mathrm{b})$. By varying the delay time t 2 the population dynamics of the indicated peaks is monitored (right panels).

In contrast the $\mathrm{CP}$ signal monitors secondary $\mathrm{BCl}_{\mathrm{L}} \rightarrow \mathrm{BP}_{\mathrm{L}} \mathrm{CS}$ on a sub-picosecond timescale (e.g. crosspeaks $\mathrm{BCl}_{\mathrm{L}, \mathrm{ESA}}$ and $\mathrm{BP}_{\mathrm{L} / \mathrm{M}, \mathrm{ESA} 2}$ ) and suppresses dominant 3 ps contributions. The unique pulse configuration is ideally suited for the investigation of $\mathrm{RC}$, where the spatial configuration of the special pair and the accessory $\mathrm{BP}_{\mathrm{L}}$ imposes nearly orthogonal exciton transition dipoles allowing to obtain complementary information from the AP and CP signal. Rotational averaging guarantees highest intensities for the $\mathrm{CP}$ signal if the transition moments between doorway and window wavepackets have orthogonal orientation. The unique arrangement of chromophores in the active L branch almost perfectly meets this requirement.

\section{Acknowledgements}

The support of the Chemical Sciences, Geosciences and Biosciences Division, Office of Basic Energy Sciences, Office of Science, U.S. Department of Energy is gratefully acknowledged. We also gratefully acknowledge the support of the National Science Foundation through Grants CHE1058791 and CHE- 0840513. B.P.F. gratefully acknowledges support from the Alexander-vonHumboldt Foundation through the Feodor-Lynen program.

\section{References}

1. D. Abramavicius, B. Palmieri, D. Voronine, F. Sanda, S. Mukamel, Chem. Rev. 109, 2350 (2009).

2. D. Abramavicius, S. Mukamel, J. Chem. Phys. 133, 184501 (2010).

3. N. S. Ginsberg, J. A. Davis, M. Ballottari, Y.-C. Cheng, R. Bassi, G. R. Fleming, Proc. Natl. Acad. Sci. U.S.A. 108, 3848 (2011). 